Artículo

\title{
Identificación y caracterización morfológica de agaves en sistemas agroforestales con metepantle en tierras campesinas
}

\author{
Rosa María García Núñez ${ }^{1}$ \\ Miriam Galán Reséndiz ${ }^{1 \S}$ \\ Jesús Axayacatl Cuevas Sánchez ${ }^{2}$ \\ Rogelio Álvarez Hernández ${ }^{3}$ \\ ${ }^{1}$ Maestría en Ciencias en Agroforestería para el Desarrollo Sostenible-Universidad Autónoma Chapingo. \\ Carretera Federal México-Texcoco km 38.5, Texcoco, México. CP. 56230. (blondynunez@gmail.com). \\ ${ }^{2}$ Departamento de Fitotecnia-Universidad Autónoma Chapingo. Carretera Federal México-Texcoco km \\ 38.5, Texcoco, México. CP. 56230. (jaxayacatl@gmail.com). ${ }^{3}$ Preparatoria Agrícola-Universidad \\ Autónoma Chapingo. Carretera Federal México-Texcoco km 38.5, Texcoco, México. CP. 56230. (roger- \\ ewl_uach@yahoo.com.mx).
}

${ }^{\S}$ Corresponding author: miriamgalanr.suelos@gmail.com.

\section{Resumen}

Se identificaron las variedades de agave en 11 clases de tierras campesinas en Tepetlaoxtoc de Hidalgo, Estado de México; así como, su caracterización morfológica de lo que popularmente se conocen como magueyes pulqueros; a través, del análisis de conglomerados y del componente principal. Se encontraron 7 variedades: Manso (Agave salmiana Otto ex Salm.), Verde (Agave americana L.), Ayoteco (Agave salmiana Otto ex Salm.) Carrizo (Agave mapisaga Trel.) y Púa larga (Agave sp.). Las variedades de maguey Maso y Verde se encuentran en todas las clases de tierras campesinas, induciendo a que todos los individuos evaluados son fenotípicamente similares o muy emparentados; en contraste, el Púa larga se encontró solamente en tres clases de tierra. Las variedades Ayoteco y Carrizo, se encontraron en cinco clases de tierra, ambas con características fenotípicas muy emparentadas con el maguey Manso. El mayor desarrollo morfológico se presentó en la clase de tierra amarilla, donde el maguey Verde, Púa larga, Carrizo y el Ayoteco obtuvieron su mejor desarrollo en diámetro de copa y altura. El maguey Manso presentó un diámetro de copa (distancia entre penca y penca abierta) de entre 1.15 a $3.5 \mathrm{~m}$ y una altura de 1.2 . a $2.8 \mathrm{~m}$. en contraste, el Carrizo presentó de 1.7 a $2.37 \mathrm{~m}$ de diámetro de copa y una altura de 1.23 a $2.1 \mathrm{~m}$ respectivamente. Los campesinos de Tepetlaoxtoc han conservado esta diversidad de variedades de maguey pulquero por la diversidad de usos múltiples que les proporcionan y continúan en su proceso de domesticación incidiendo en su morfología.

Palabras clave: agroforestería, maguey pulquero, tierras campesinas.

Recibido: marzo de 2020

Aceptado: mayo de 2020 


\section{Introducción}

En los sistemas agroforestales mesoamericanos el empleo del metepantle, concepto formado por los vocablos del náhuatl «metl», maguey o agave, y «pantli», bandera o división (Montemayor, 2009), es una práctica agrícola ancestral, que hace referencia al cultivo de maguey en hileras perpendiculares a la pendiente del terreno. En el municipio de Tepetlaoxtoc de Hidalgo, Estado de México, esta práctica agrícola ancestral es vigente, porque el cultivo del agave pulquero, persiste en las condiciones ecológicas de la región, reflejado en prácticas sociales y productivas determinadas por la articulación histórica de sus procesos tecnológicos y culturales, así como su impacto en la región, privilegiada como centro de origen y diversidad del agave por selección natural desde hace 9000 años, por la domesticación, manejo y usos múltiples que los pueblos originarios han desarrollado. Sin embargo, al mismo tiempo es una estrategia para la conservación de la biodiversidad y la seguridad alimentaria de las comunidades (Koohafkan y Altieri, 2011; Flores-Sánchez et al., 2012).

El cultivo y cultura del agave pulquero, es una muestra del manejo complejo de los recursos suelo y vegetación; conocimientos ancestrales, que se han conservado con ligeras modificaciones en sus nombres o readaptados a las innovaciones tecnológicas (Aguilar et al., 2014). El producto del maguey, desde la época prehispánica, más renombrado es el pulque, en náhuatl «octli», la bebida esencial en la vida ritual como bebida en las ceremonias relacionadas con diferentes deidades como Quetzalcóatl y Mayahuel; además estar presente en innumerables símbolos, mitos, tradiciones, imágenes, sonidos, texturas, hábitats, alimentos, vestimentas, juegos y lenguaje coloquial (Olivier, 2012). Sin embargo, estos sistemas agrícolas no necesariamente son productivos, debido a la problemática social-ambiental detonada a partir del cambio en el uso y manejo de los recursos naturales y del hábito de consumo de las comunidades campesinas, aunada a un deterioro de la capacidad productiva de la tierra, que ha fracturado, la relación milenaria hombre-agave.

En la búsqueda de generar estrategias conjuntas de caracterización, revaloración y mejoramiento de esta práctica agrícola ancestrales de manejo de la tierra con metepantle, en Tepetlaoxtoc, el objetivo de este trabajo fue identificar las variedades de agave pulquero y su estructura morfológica, con base en el conocimiento científico y popular, que permitirán su posterior manejo integral, reconociendo su utilidad para conservar la agrodiversidad de las comunidades que lo practican, ya que junto con el cultivo del maíz, fue fundamental para la vida de los pueblos del centro de México a lo largo de su historia, por las condiciones de cultivos entre hilera, su forma de aprovechamiento, sus procesos productivos y la cultura del agave (Godoy et al., 2003).

\section{Materiales y métodos}

\section{Descripción geográfica del área de estudio}

El área de estudio en Tepetlaoxtoc, se ubica entre las coordenadas $19^{\circ} 35^{\prime} 54^{\prime \prime}$ y $19^{\circ} 24^{\prime} 30^{\prime \prime}$ latitud norte, $98^{\circ} 50^{\prime} 02^{\prime \prime}$ y $98^{\circ} 46^{\prime} 34^{\prime \prime}$ longitud oeste y una elevación entre 2300 y 2469 m, en una diversidad de estructuras geológicas del proceso volcánico-tectónico comprendido entre el final del periodo Paleozoico y durante el Mesozoico, que resultó en paisajes de lomeríos y planos aluviales (Gutiérrez et al., 2017). Pertenece a la cuenca Pánuco, subcuenca río Moctezuma, con las corrientes intermitentes río Hondo y río Sila (INEGI, 2010). 
El clima corresponde al tipo Bs(c)wk'g, de acuerdo con la clasificación de Köppen, modificado por García, (1973), con temperatura media anual de $15.5^{\circ} \mathrm{C}$, máxima de $26.9^{\circ} \mathrm{C}$ y una mínima de $3.9^{\circ} \mathrm{C}$. Los suelos reportados son Phaeozem, Vertisol (Gutiérrez et al., 2017), Andosol y Umbrisol (INEGI, 2010), en donde se cultiva agave pulquero en hileras perpendiculares a la pendiente del terreno y principalmente maíz, frijol, calabaza y haba entre hileras bajo agricultura de temporal con algunos cultivos introducidos como la avena.

\section{Variedades de agave pulquero}

La caracterización se realizó en un total de 33 unidades muéstrales distribuidas en las 11 clases de tierra campesina: tepetatuda roja (tr), tepetatuda parda (tp), barro blanco (bb), barro pardo (bp), tepetatuda blanca (tb), barro oscuro (bo), amarilla (am), barro (ba), amarilla arenosa (aa), tepetatuda amarilla (tm) y amarilla barruda (ab). Las cuales fueron nombradas en términos que los campesinos usan cotidianamente para referirse a ellas respecto a ciertos rasg os de la capa arable del suelo.

Como el color, textura, consistencia en húmedo, consistencia en seco, retención de humedad, oportunidad para el laboreo y fertilidad del suelo, que ocurren sobre suelos de clase Regosols, Anthrosols (terrazas) y Fluvisols (plano aluvial). Los nombres comunes de las variedades de agave pulquero presentes y la edad se determinaron en campo en compañía de los informantes clave, y la identificación taxonómica se obtuvo de acuerdo con la base de datos de nombres técnicos o de uso común en el aprovechamiento de los agaves en México, sujeta al Código Internacional de Nomenclatura Botánica (Colunga-García, 2006) y a su identificación en herbario.

\section{Estructura morfológica de las variedades de agave pulquero}

Se evaluaron caracteres morfológicos establecidos en la guía técnica para la descripción varietal de Agave sp., de la Secretaría de Agricultura, Ganadería, Desarrollo Rural, Pesca y Alimentación (SAGARPA) y del Servicio Nacional de Inspección y Certificación de Semillas (SNICS)- (2014), con un muestreo a conveniencia, en las parcelas que los informantes clave consideraron representativas de los sistemas agroforestales con metepantle, debido a que las clases de tierra poseen más usos, urbano, minero, pecuario.

Los transectos fueron de 30 metros de largo, perpendiculares a la pendiente del terreno, con un ancho que dependió de la distancia más grande del diámetro de penca abierta del maguey más ancho del transecto, además se dejaron $2 \mathrm{~m}$ al inicio de cada transecto para evitar el efecto orilla $\mathrm{y}$ la elección aleatoria de tres plantas por transecto. A cada planta se le evaluó, altura (m), número total pencas (hojas desplegadas), área basal (m, \%), diámetro de copa, que para fines prácticos se nombró al diámetro de penca a penca abierta, $(\mathrm{m}, \%)$, la cobertura $(\mathrm{m}, \%)$ en dos orientaciones N$\mathrm{S}$ y E-O.

La morfología de las hojas se evaluó mediante la elección aleatoria de tres pencas por planta, a las que se les midió, longitud (m), ancho de la parte media de la hoja (m), número de espinas laterales, longitud de la espina terminal $(\mathrm{mm})$ y la separación entre espinas laterales $(\mathrm{mm})$ con el uso del vernier. Además, se cuantificó la densidad de plantación (número de agaves/variedad/transecto). 


\section{Evaluación cuantitativa de las variedades de agave pulquero en las clases campesinas de tierra}

Este análisis se basó en la distancia Euclidiana; a partir, de las características morfológicas, evaluadas por cada variedad de agave pulquero en las 11 clases de tierras campesinas, que permitió distinguir los conglomerados con el uso del programa estadístico Statgraphics Centurion ${ }^{\circledR}$ XVI versión 1.11 .

Se aplicó el análisis de conglomerados (análisis clúster) y el análisis del componente principal con base en la metodología sugerida por Dallas (2004) para evaluar en forma cuantitativa los niveles jerárquicos que sirvió como base para clasificar la estructura morfológica de las variedades de agave pulquero, de tal manera que la distribución de los valores de las variables fuera lo más homogénea y entre conglomerados los más distintos entre sí.

\section{Resultados y discusión}

\section{Variedades de agave pulquero}

Se diferenciaron las variedades que popularmente se conocen como maguey Manso, maguey Verde, maguey Ayoteco, maguey Carrizo, maguey púa larga, que se encuentran en el grupo de las especies del género Agave reportadas para la elaboración de bebidas fermentadas y destiladas (Gentry, 1982). De acuerdo a estudios taxonómicos (Rzedowski y Rzedowski, 2005), tanto los magueyes Manso, como Ayoteco se clasifican como Agave salmiana, mientras que a Carrizo como Agave mapisaga Trel (Alfaro, 2007).

La distribución de las variedades de agave pulquero en las clases de tierras campesinas se muestra en el Cuadro 1. Los magueyes Manso y Verde estuvieron presentes en todas las clases de tierra, el Ayoteco se encontró en las clases tb, tm, aa, am. ba y ab, el Carrizo en las clases tb, tm, aa, ba y bb y la púa larga solamente en las clases tr, aa y am.

Cuadro 1. Variedades de agave pulquero presentes en las clases de tierra campesina en Tepetlaoxtoc, Estado de México.

\begin{tabular}{ccc}
\hline Clase de tierra & Abreviatura & Variedades de agave pulquero \\
\hline Tepetatuda roja & tr & 1,2 y 5 \\
Tepetatuda parda & tp & 1 y 2 \\
Tepetatuda barruda & tb & $1,2,3$ y 4 \\
Tepetatuda Amarilla & tm & $1,2,3$ y 4 \\
Amarilla arenosa & aa & $1,2,3,4$ y 5 \\
Amarilla & am & $1,2,3$ y 5 \\
Barro pardo & bp & 1 y 2 \\
Barro & ba & $1,2,3$ y 4 \\
Barro obscuro & bo & 1 y 2 \\
Amarilla barruda & ab & 1,2 y 3 \\
Barro blanco & bb & 1,2 y 4 \\
\hline
\end{tabular}

1= maguey Manso; 2= maguey Verde; 3= maguey Ayoteco; 4= maguey Carrizo; y 5= maguey púa larga. 
Los agaves pulqueros A. salmiana Otto ex Salm. y A. americana L. que se encuentran presentes en todas las clases de tierra, de acuerdo con Colunga-García (2006) se encuentran en el grupo de los magueyes que siguen teniendo relevancia regional y potencial, que se han cultivado desde la época prehispánica y que actualmente son objeto de más difusión (Álvarez et al., 1998; Valadez, 2014; Vázquez et al., 2016; Vela, 2018).

Autores como García-Mendoza (1995) reporta que al agave pulquero se desarrolla en una amplia variedad de suelos, en el área de estudio se observó que van desde las tierras de tipo tepetatuda hasta las de tipo barro, cuya etiqueta corresponde a una terminología descriptica que involucra los criterios de color, textura y presencia de tepetate, en relación tanto a características de oportunidad para el laboreo como de las peculiaridades del diseño del metepantle.

Investigaciones realizadas por Alfaro et al. (2007) indican que este tipo de distribución son producto de selecciones específicas de los productores en la búsqueda de obtener materiales morfológicos definidos y diferenciados relacionados con las características de calidad y cantidad del aguamiel, entre muchas otras, ya que originan nuevos fenotipos con una mayor capacidad de adaptación y respuesta a ambientes extremos, lo que puede contribuir al éxito en la naturaleza o en su selección y uso en la agricultura (García-Mendoza, 2011).

La presencia de dos tipos de A. salmiana (Manso y Ayoteco) se debe a su plasticidad; es decir, infiltración genética de las formas cultivadas con silvestres (García-Mendoza, 2011) y la variabilidad morfológica es debida la intervención humana (Mora-López et al., 2011). Debido a esta antigua e intensa relación entre los humanos y el agave hay variantes con importancia regional, nacional e internacional, de las cuales existe un conocimiento ancestral considerable y una variación morfológica alta (Colunga-García et al., 1996).

\section{Estructura morfológica de las variedades de agave pulquero}

Desde el punto de vista morfológico las variedades de agave pulquero encontradas son diferentes (Cuadro 2); sin embargo, de acuerdo con Alfaro et al. (2007) genéticamente son semejantes el maguey maso y el Ayoteco, ya que son selecciones que se han originado a partir de un ancestro común y que en algún momento se separaron y dieron origen a las especies que actualmente se conocen.

Cuadro 2. Estructura morfológica de las variedades de maguey en las clases de tierra campesina en Tepetlaoxtoc, México.

\begin{tabular}{cccccccccc}
\hline Variedades & Clase de tierra & $\mathrm{E}$ & $\mathrm{DC}$ & $\mathrm{C}$ & $\mathrm{C} \%$ & $\mathrm{Db}$ & $\mathrm{Ab}$ & $\mathrm{Ab} \%$ & $\mathrm{~A}$ \\
\hline Manso & $\mathrm{tr}$ & 5 & 1.79 & 2.52 & 4.66 & 0.5 & 0.19 & 0.36 & 1.38 \\
& $\mathrm{tp}$ & 8 & 3.5 & 9.62 & 8.91 & 0.8 & 0.5 & 0.47 & 1.92 \\
& $\mathrm{tb}$ & 12 & 3.25 & 8.3 & 6.91 & 1.25 & 1.23 & 1.06 & 2.8 \\
& $\mathrm{tm}$ & 15 & 2.12 & 3.53 & 5.47 & 0.77 & 0.46 & 0.71 & 2.2 \\
& $\mathrm{aa}$ & 16 & 3.2 & 8.02 & 8.35 & 0.42 & 0.14 & 0.14 & 1.22 \\
& $\mathrm{am}$ & 5 & 1.26 & 1.25 & 1.26 & 0.61 & 0.29 & 0.3 & 1.65 \\
& $\mathrm{bp}$ & 4 & 1.15 & 1.04 & 2.47 & 0.4 & 0.12 & 0.3 & 1.45 \\
\hline
\end{tabular}




\begin{tabular}{|c|c|c|c|c|c|c|c|c|c|}
\hline Variedades & Clase de tierra & $\mathrm{E}$ & $\mathrm{DC}$ & $\mathrm{C}$ & $\mathrm{C} \%$ & $\mathrm{Db}$ & $\mathrm{Ab}$ & $\mathrm{Ab} \%$ & A \\
\hline \multirow{12}{*}{ Verde } & ba & 9 & 1.44 & 1.63 & 2.52 & 0.27 & 0.06 & 0.09 & 1.6 \\
\hline & bo & 9 & 1.85 & 2.69 & 4.07 & 0.62 & 0.3 & 0.46 & 1.4 \\
\hline & $a b$ & 10 & 1.95 & 2.99 & 4.33 & 0.53 & 0.22 & 0.31 & 1.6 \\
\hline & $\mathrm{bb}$ & 8 & 1.55 & 1.89 & 3.14 & 0.48 & 0.18 & 0.3 & 1.4 \\
\hline & $\operatorname{tr}$ & 5 & 1.6 & 2.01 & 3.72 & 0.46 & 0.17 & 0.31 & 1.11 \\
\hline & tp & 10 & 2.53 & 5.01 & 6.42 & 0.34 & 0.09 & 0.12 & 2.78 \\
\hline & $\mathrm{tb}$ & 10 & 2.62 & 5.37 & 5.77 & 0.69 & 0.37 & 0.4 & 2.25 \\
\hline & $\mathrm{tm}$ & 15 & 2.01 & 3.17 & 4.92 & 0.4 & 0.12 & 0.19 & 2.1 \\
\hline & aa & 7 & 3.09 & 7.47 & 8.04 & 0.37 & 0.1 & 0.12 & 2.1 \\
\hline & am & 7 & 2 & 3.14 & 2.99 & 0.65 & 0.33 & 0.32 & 2.3 \\
\hline & bp & 5 & 1.75 & 2.41 & 3.82 & 0.5 & 0.19 & 0.31 & 1.26 \\
\hline & ba & 10 & 1.79 & 2.52 & 4.3 & 0.49 & 0.18 & 0.32 & 1.75 \\
\hline \multirow{7}{*}{ Ayoteco } & bo & 15 & 1.69 & 2.24 & 3.94 & 0.55 & 0.24 & 0.42 & 1.7 \\
\hline & $a b$ & 10 & 1.83 & 2.62 & 4.71 & 0.51 & 0.2 & 0.37 & 2 \\
\hline & $\mathrm{bb}$ & 7 & 1.44 & 1.62 & 3.59 & 0.34 & 0.09 & 0.2 & 1.2 \\
\hline & $\mathrm{bb}$ & 8 & 3 & 7.07 & 7.6 & 0.47 & 0.17 & 0.18 & 1.71 \\
\hline & $\mathrm{tm}$ & 15 & 1.96 & 3.02 & 4.68 & 0.42 & 0.14 & 0.21 & 2 \\
\hline & aa & 7 & 2.78 & 6.05 & 6.5 & 0.26 & 0.05 & 0.06 & 2.03 \\
\hline & am & 7 & 2.04 & 3.27 & 3.11 & 0.59 & 0.27 & 0.26 & 1.77 \\
\hline \multirow{5}{*}{ Carrizo } & ba & 9 & 1.65 & 2.13 & 3.3 & 0.33 & 0.09 & 0.13 & 1.64 \\
\hline & $a b$ & 9 & 1.81 & 2.57 & 4.64 & 0.53 & 0.22 & 0.39 & 1.67 \\
\hline & $\mathrm{bb}$ & 5 & 2.37 & 4.41 & 4.74 & 0.69 & 0.37 & 0.4 & 1.93 \\
\hline & $\mathrm{tm}$ & 10 & 1.42 & 1.57 & 3.49 & 0.23 & 0.04 & 0.09 & 2.1 \\
\hline & aа & 7 & 2.59 & 5.27 & 5.49 & 0.39 & 0.12 & 0.12 & 1.45 \\
\hline \multirow{5}{*}{ Púa larga } & ba & 10 & 1.7 & 2.26 & 3.86 & 0.52 & 0.21 & 0.36 & 1.54 \\
\hline & $\mathrm{tb}$ & 4 & 1.71 & 2.3 & 5.89 & 0.34 & 0.09 & 0.23 & 1.25 \\
\hline & $\operatorname{tr}$ & 5 & 1.75 & 2.41 & 4.45 & 0.47 & 0.17 & 0.32 & 1.3 \\
\hline & aa & 7 & 2.59 & 5.27 & 5.49 & 0.39 & 0.12 & 0.12 & 1.45 \\
\hline & am & 5 & 1.15 & 1.04 & 1.05 & 0.66 & 0.34 & 0.34 & 2.15 \\
\hline
\end{tabular}

$\mathrm{E}=$ edad de la planta (años); $\mathrm{DC}=$ diámetro de copa $(\mathrm{m}) ; \mathrm{C}=$ cobertura $\left(\mathrm{m}^{2}\right) ; \mathrm{C} \%=$ cobertura $(\%) ; \mathrm{Db}=$ diámetro basal $(\mathrm{m}) ; \mathrm{Ab}=$ área basal $\left(\mathrm{m}^{2}\right) ; \mathrm{Ab} \%=$ área basal $(\%) ; \mathrm{A}=$ altura $(\mathrm{m})$.

El maguey Manso presentó una variación en el diámetro de copa (de penca a penca abierta) de 1.15 a $3.5 \mathrm{~m}$, con una cobertura de 1.04 a $9.62 \mathrm{~m}$ y el diámetro basal de 0.27 a $1.25 \mathrm{~m}$, el área basal fue de 0.06 a $1.23 \mathrm{~m}^{2}$ y una altura de 1.22 a $2.8 \mathrm{~m}$. Sin embargo, en la clase de tierra tepetatuda parda fue donde presentó el mayor diámetro de copa $(3.5 \mathrm{~m})$ a una edad de 8 años y una altura de 1.92, en contraste a la misma edad en la clase barro blanca presentó el menor desarrollo (1.55 m y $1.4 \mathrm{~m}$ respectivamente). 
Estas características de altura de la planta, área basal, número de hojas y longitud de hojas son de particular importancia para la obtención de aguamiel y la obtención de penca para barbacoa (platillo regional) (Alfaro et al., 2007). El maguey Verde se encontró en todas las clases campesinas de tierra, siendo en la clase amarilla donde presentó el diámetro de copa más grande (3.09 m) a una edad de 7 años y una altura de $2.1 \mathrm{~m}$, en contraste en el barro blanco presentó un diámetro de copa de $1.44 \mathrm{~m}$ y una altura de $1.2 \mathrm{~m}$ a la misma edad.

El maguey Ayoteco presentó edades de plantación de 7 a 15 años, la clase de tierra donde se presentó el diámetro de copa más grande $(3 \mathrm{~m})$ fue barro blanco; sin embargo, la mayor altura se encontró en la amarilla $(2.03 \mathrm{~m})$ en una edad de 7 años. La relación entre altura de planta y largo de hojas, indica que a mayor altura se espera una mayor longitud de hojas, de manera similar con lo reportado por Alfaro et al. (2007).

El maguey Carrizo se encontró en plantaciones con edades de 4 a 10 años, alcanzando su mayor altura en tepetatuda amarilla $(2.2 \mathrm{~m})$ y el diámetro de copa máximo de $2.59 \mathrm{~m}$ en la clase de tierra amarilla a una edad de 7 años. En el púa larga, se observaron edades de plantación de 5 a 7 años y alturas de 1.3 a $2.25 \mathrm{~m}$, con diámetros de copa de 1.15 a $2.41 \mathrm{~m}$, coberturas de copa de 1.04 a 2.41 $\mathrm{m}^{2}$, diámetro basal de 0.39 a $0.66 \mathrm{~m}$ con un número de pencas desplegadas de 10 a 18 . La clase de tierra campesina donde presentó su mejor desarrollo morfológico fue la amarilla (Cuadro 3).

Cuadro 3. Estructura morfológica la hoja de las variedades de maguey en las clases de tierra campesina en Tepetlaoxtoc, México.

\begin{tabular}{|c|c|c|c|c|c|c|c|}
\hline Variedad & Clase de tierra & $\mathrm{NTH}$ & $\mathrm{Lh}$ & Aph & LET & NEL & SEL \\
\hline \multirow[t]{11}{*}{ Manso } & $\operatorname{Tr}$ & 18 & 1.15 & 0.2 & 73.1 & 10 & 29.95 \\
\hline & $\mathrm{Tp}$ & 17 & 1.22 & 0.19 & 64.92 & 33 & 57.2 \\
\hline & $\mathrm{Tb}$ & 20 & 1.79 & 0.3 & 53.5 & 50 & 40.5 \\
\hline & $\mathrm{Tm}$ & 22 & 1.89 & 0.25 & 53 & 44 & 49.1 \\
\hline & $\mathrm{Aa}$ & 13 & 2 & 0.32 & 74.7 & 40 & 68.29 \\
\hline & $\mathrm{Am}$ & 12 & 1.1 & 0.27 & 62.92 & 30 & 60.42 \\
\hline & $\mathrm{Bp}$ & 10 & 0.94 & 0.22 & 76.3 & 33 & 45.5 \\
\hline & $\mathrm{Ba}$ & 10 & 1.1 .1 & 0.22 & 80.9 & 30 & 66.6 \\
\hline & Bo & 14 & 1.3 & 0.26 & 60.37 & 20 & 62.05 \\
\hline & $\mathrm{Ab}$ & 15 & 1.57 & 0.23 & 81.33 & 19 & 75.57 \\
\hline & $\mathrm{Bb}$ & 18 & 1.15 & 0.2 & 73.1 & 10 & 29.95 \\
\hline \multirow[t]{8}{*}{ Verde } & $\operatorname{Tr}$ & 12 & 1.09 & 0.16 & 77.11 & 28 & 38.8 \\
\hline & $\mathrm{Tp}$ & 12 & 1.09 & 0.17 & 77.11 & 28 & 38.8 \\
\hline & $\mathrm{Tb}$ & 19 & 1.33 & 0.3 & 51.29 & 44 & 70.5 \\
\hline & $\mathrm{Tm}$ & 20 & 1.79 & 0.31 & 53.5 & 50 & 40.5 \\
\hline & $\mathrm{Aa}$ & 12 & 1.09 & 0.17 & 77.11 & 28 & 38.8 \\
\hline & Am & 11 & 1.1 & 0.23 & 81.4 & 27 & 79.7 \\
\hline & $\mathrm{Bp}$ & 11 & 0.93 & 0.18 & 58.88 & 30 & 46.42 \\
\hline & $\mathrm{Ba}$ & 12 & 1.08 & 0.22 & 76.7 & 25 & 55.4 \\
\hline
\end{tabular}




\begin{tabular}{cccccccc}
\hline Variedad & Clase de tierra & NTH & Lh & Aph & LET & NEL & SEL \\
\hline \multirow{6}{*}{ Ayoteco } & Bo & 11 & 1.1 & 0.23 & 79.1 & 27 & 78.7 \\
& $\mathrm{Ab}$ & 11 & 1.1 & 0.23 & 79.1 & 27 & 78.7 \\
& $\mathrm{Bb}$ & 11 & 1.1 & 0.23 & 79.1 & 27 & 78.7 \\
& $\mathrm{Bb}$ & 19 & 1.33 & 0.3 & 51.29 & 44 & 70.2 \\
& $\mathrm{Tm}$ & 20 & 1.79 & 0.31 & 53.5 & 50 & 40.5 \\
& $\mathrm{Aa}$ & 10 & 1.1 .1 & 0.22 & 80.9 & 31 & 66.6 \\
& $\mathrm{Am}$ & 12 & 1.1 & 0.27 & 62.92 & 30 & 60.42 \\
& $\mathrm{Ba}$ & 15 & 1.58 & 0.21 & 74.59 & 38 & 44.12 \\
& $\mathrm{Ab}$ & 17 & 1.22 & 0.19 & 64.92 & 33 & 57.2 \\
& $\mathrm{Bp}$ & 18 & 1.49 & 0.31 & 63.07 & 39 & 71.63 \\
& $\mathrm{Tm}$ & 20 & 2.32 & 0.38 & 57.68 & 44 & 42.94 \\
& $\mathrm{Aa}$ & 14 & 0.89 & 0.21 & 67.3 & 31 & 44.4 \\
& $\mathrm{Ba}$ & 16 & 1.1 & 0.23 & 79.1 & 27 & 78.7 \\
& $\mathrm{~Tb}$ & 15 & 1 & 0.23 & 84.53 & 22 & 87.44 \\
& $\mathrm{Tr}$ & 18 & 1.15 & 0.2 & 73.1 & 10 & 29.95 \\
& $\mathrm{Aa}$ & 13 & 2 & 0.32 & 74,7 & 40 & 68.29 \\
& $\mathrm{Am}$ & 10 & 1.1 & 0.23 & 79.1 & 27 & 78.7 \\
\hline
\end{tabular}

$\mathrm{NTH}=$ número total de hojas desplegadas; $\mathrm{Lh}=$ longitud de la hoja $(\mathrm{m}) ; \mathrm{Aph}=$ ancho parte media de la hoja $(\mathrm{m}) ; \mathrm{LET}=$ longitud de la espina terminal $(\mathrm{mm}) ; \mathrm{NEL}=$ número de espinas laterales; $\mathrm{SEL}=$ separación entre espinas laterales $(\mathrm{mm})$.

Los valores medios de las características morfológicas de la hoja del maguey Manso mostraron una longitud de 0.94 a $2 \mathrm{~m}$, la parte media de la hoja varió 0.19 a $0.32 \mathrm{~m}$, la longitud de la espina terminal de 53 a $81.33 \mathrm{~mm}$, el número de espinas laterales de 10 a 50 y la separación entre espinas laterales de 29.95 a $75.57 \mathrm{~mm}$. mientras que la longitud de las hojas del maguey Verde varió de 0.93 a $1.79 \mathrm{~m}$, la parte media de la hoja de 0.16 a $0.31 \mathrm{~m}$, la longitud de la espina terminal de 51.29 a $81.4 \mathrm{~mm}$.

El número de espinas laterales tuvo un intervalo de 25 a 50 con una separación laterales de 40.5 a $79.7 \mathrm{~mm}$. Esto se atribuye al efecto del ambiente sobre el fenotipo de las plantas, ya que las variantes Verde y Manso son genéticamente muy diferentes, pero fenotípicamente semejantes (Alfaro et al., 2007). El maguey Ayoteco presentó un número de pencas desplegadas de 10 a 20, con una longitud de 1.1 A $1.79 \mathrm{~m}$, la parte media de la hoja de 0.19 a $0.31 \mathrm{~m}$, la longitud de la espina terminal de 51.29 y $80.9 \mathrm{~mm}$, el número de espinas laterales de 30 a 50 y la separación entre espinas laterales de 40.5 a $79.7 \mathrm{~mm}$.

En agaves se ha documentado que uno de los elementos del síndrome de domesticación es el gigantismo (Colunga-García et al., 1996), los resultados del estudio de Mora-López et al. (2011) indican que la variedad Ayoteco presenta rosetas grandes superiores a los tres metros de altura. Las plantas más altas de maguey pulquero fueron también las que presentaron más hojas, hojas más largas y con más espinas laterales. Los caracteres morfológicos evaluados no permitieron establecer la relación genética existente entre Carrizo y Verde ya que en campo estos magueyes son muy diferentes entre sí tal como menciona Alfaro et al. (2007). 
La variedad de maguey púa larga se localizó solamente en las tierras tr, aa y am, con un número total de pencas desplegadas de 10 a 18 , longitud de hoja de 1.1 a $2 \mathrm{~m}$ con ancho de parte media con un intervalo de 0.2 a $0.32 \mathrm{~m}$, número de espinas de 10 a 40 y separación entre espinas de 29.95 a $8.7 \mathrm{~mm}$. Las características morfológicas que permitieron distinguir mejor a las especies y variantes de magueyes pulquero fueron la longitud de la espina principal y el número de espinas laterales. Las plantas más altas de maguey pulquero son las que presentan mayor número de hojas, hojas más largas y mayor número de espinas laterales.

Las plantas que presentan mayor altura también son las que muestran un mayor número de hojas, como en el caso de maguey Manso, que concuerdan con los resultados de Rzedowski y Rzedowski (2005) ya que ambos son Agave salmiana. La caracterización morfológica contribuye a realizar la protección legal de variedades o cultivares de uso común de este tipo de agave endémico de México, y con ello promover su conservación y aprovechamiento sustentable (Avendaño-Arrazate et al., 2015).

Evaluación cuantitativa de la estructura de las variedades de agave pulquero y las clases campesinas de tierra Se obtuvieron cinco dendrogramas en el análisis de conglomerados; a partir, de la estructura morfológica de cada variedad de maguey pulquero identificadas en las clases de tierras campesinas (Figura 1).

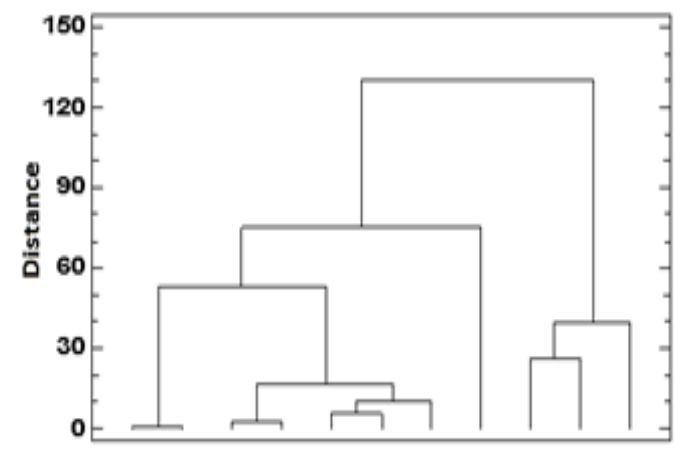

a) $\operatorname{Tr} B b$ Am Bo Bp Ba Ab Aa Tp Tm Bp

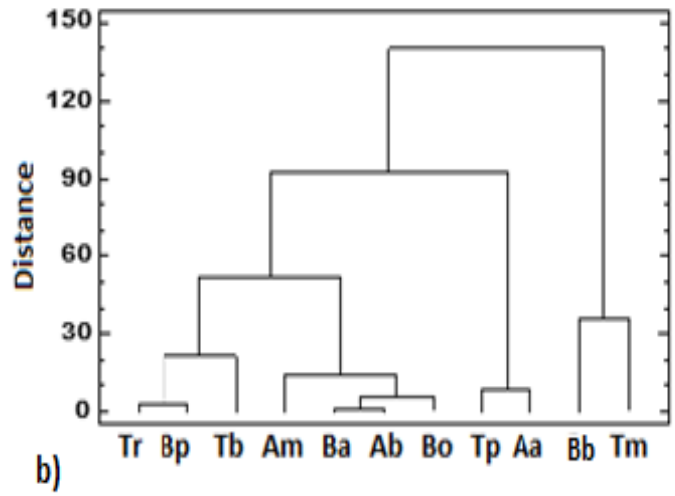

b)
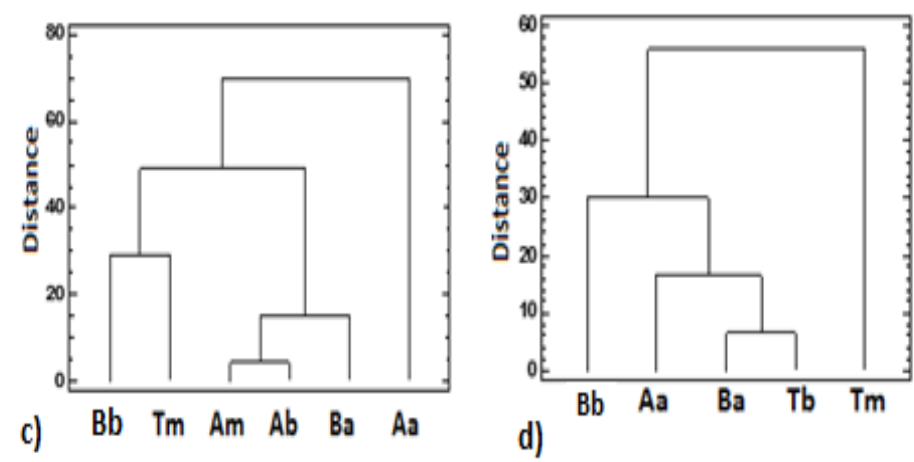

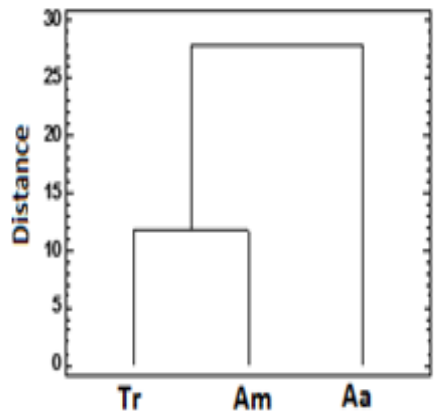

e)

Figura 1. Dendrograma del análisis de conglomerados de la estructura morfológica del maguey a) Manso; b) Verde; C) Ayoteco; d) Carrizo; y e) Púa larga en las clases de tierra campesina donde se distribuyen en Tepetlaoxtoc, Estado de México. 
Se evaluaron cuantitativamente respecto a las propiedades de la estructura general de la planta y particular de la hoja que resultó una alternativa para diferenciar y agrupar las clases de tierra en donde los agaves poseen características similares, lo que permitirá definir el potencial agrícola ya que, como mencionan Alfaro et al. (2007), ambas se complementan. Bowley (1999) enfatiza que detectar las clases de tierra con mayor importancia en la diferenciación de los grupos generados es de mucha utilidad ya que se ve la adaptación en diferentes clases.

Los magueyes Manso y Verde se distribuyen en todas las clases debido a que todos los individuos evaluados son fenotípicamente iguales o muy emparentados, en contraste, con el maguey Ayoteco y el Carrizo se observaron en 5 clases, por lo que ambas variedades botánicas poseen características fenotípicas muy emparentadas con el maguey Manso, en contraste hay menos cercanía con las características del Púa larga ya que sólo se encontró en tres clases de tierras.

Por lo que es posible distinguir las selecciones generadas por los productores en el grado de asociación entre las características morfológicas de los agaves pulqueros y las clases de tierra, que de acuerdo con Gaspar y Pérez (2009) está determinado por la separación angular que forman sus proyecciones; además considera la distancia de cada variable; a partir, del origen y al proceso de humanización mencionando por Mora-López et al. (2011).

El análisis del componente principal permitió establecer que el número de espinas laterales y longitud de espina principal representan $74 \%$ de la variabilidad en la estructura morfológica de los magueyes. Además, se detectó correlación entre altura de planta y número de espinas laterales, debido a que en las plantas más altas las hojas tienden a ser más grandes y con más espinas laterales.

Mora-López et al. (2011) evaluaron 48 atributos morfológicos en la sección Salmianae, de los cuales, los coeficientes de variación más altos se presentaron en los caracteres: altura de la roseta, longitud de la penca, longitud de los dientes, diámetro de la roseta, grosor de la cutícula, número de dientes y distancia entre dientes (Figura 2).
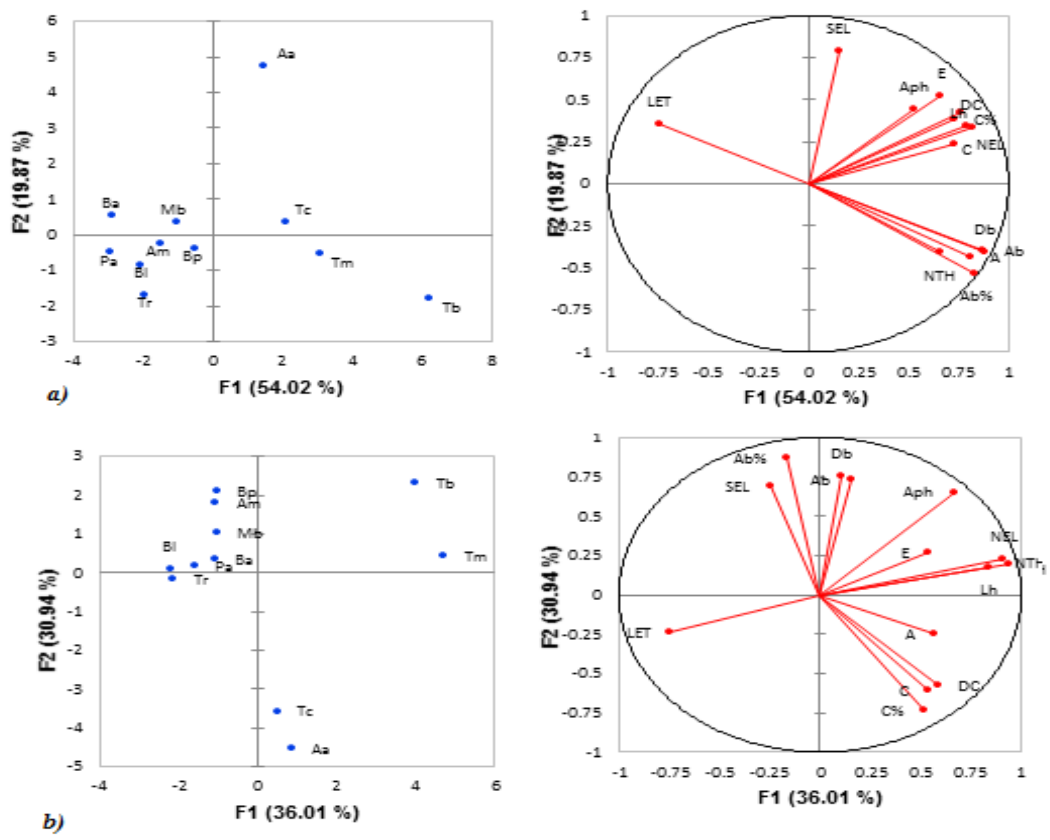

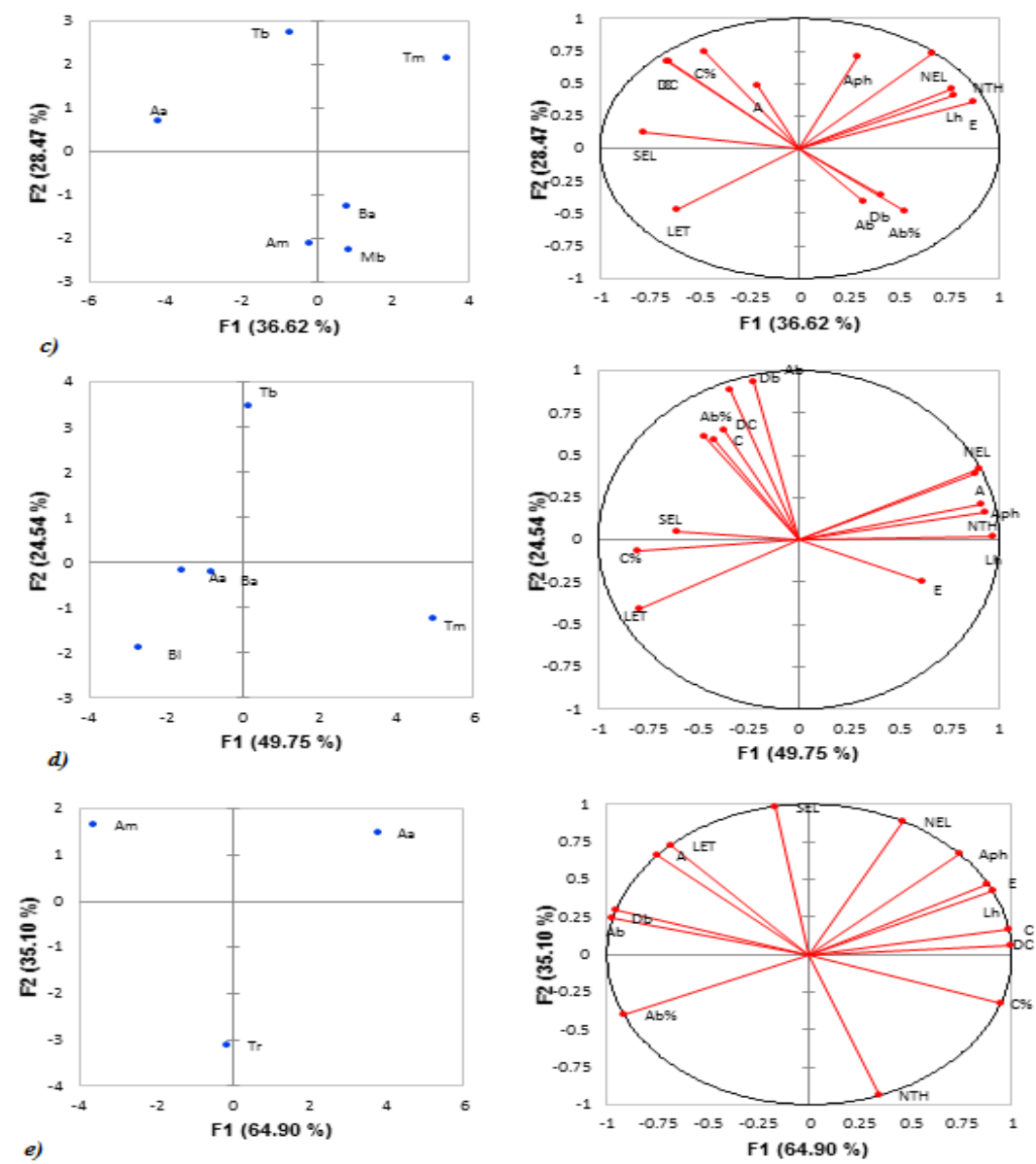

Figura 2. Análisis del componente principal de la estructura morfológica del maguey a) Manso; b) Verde; C) Ayoteco; d) Carrizo; y e) Púa Larga, en las clases de tierra campesina se distribuyen en Tepetlaoxtoc, México.

\section{Conclusiones}

Se logró identificar 5 variedades de agave pulquero y su estructura morfológica en 11 tipos de tierra campesina destacando el Manso y Verde que se encontraron en todas las clases de tierra. Sin embargo, el mayor desarrollo se presentó en la clase de tierra amarilla, donde el maguey Verde, púa larga, Carrizo y el Ayoteco obtuvieron su mejor desarrollo en diámetro de copa y altura.

Los productores de la región de Tepetlaoxtoc han seleccionado estas variedades de magueyes que les han proporcionado una diversidad de usos múltiples, los cuales tienen un grado de asociación entre las características morfológicas de los agaves pulqueros y los beneficios que les proporcionan, con ello conservan la agrodiversidad de esta comunidad que junto con el cultivo del maíz han sido fundamentales a lo largo de su historia para la vida de los pueblos del centro de México. 


\section{Literatura citada}

Aguilar, J. B.; Enríquez, D. V. R.; Rodríguez-Ortiz, G.; Granados S. D. y Cerero, B. M. 2014. El estado actual de Agave salmiana y A. mapisaga del Valle de México. Revista Mexicana de Agroecosistemas. 1(2):106-120.

Álvarez, P. A. M.; Cassiano, G. y Villa, K. A. 1998. La explotación del maguey pulquero en la zona de Metztitlan: datos etnográficos y arqueológicos. Dimensión Antropológica. 13(1):7-30.

Alfaro, R. G.; Legaria, S. J. P. y Rodríguez, P. J. E. 2007. Diversidad genética en poblaciones de agaves pulqueros (Agave spp.) del nororiente del Estado de México. Revista Fitotecnia Mexicana. 30(1):1-12.

Avendaño-Arrazate, C. H.; Iracheta-Donjuan, L.; Gódinez-Aguilar, J. C.; López-Gómez, P. y Barrios-Ayala, A. 2015. Caracterización morfológica de Agave cupreata, especie endémica de México. Phyton (Buenos Aires). 84(1):148-162.

Bowley, R. S. 1999. A Hitchhiker's Guide to Statistics in Plant Biology. First edition. Canadá: Ed. Ampersand Printing. Guelph, Ontario. 266 p.

Colunga-García, M. P.; Estrada-Loera, E. y May-Pat, F. 1996. Patterns of morphological variation, diversity, and domestication of wild and cultivated populations of Agave in Yucatan, Mexico. American Journal of Botany. 83(8):1069-1082.

Colunga-García, M. P. 2006. Base de datos de Nombres técnicos o de uso común en el aprovechamiento de los agaves en México. Centro de Investigación Científica de Yucatán AC Final Report SNIB-CONABIO. Yucatán. 206 p.

Dallas, E. J. 2004. Métodos multivariados aplicados al análisis de datos. México. Revista Mexicana de Ciencias Forestales. 6(32):17-33.

Flores-Sánchez, D.; Navarro-Garza, H.; Carballo-Carballo, A. y Pérez-Olvera, M. 2012. Sistemas de cultivo y biodiversidad periurbana: Estudio de caso en la Cuenca del Río Texcoco. Agricultura, Sociedad y Desarrollo. 9(2):209-223.

García, D. M. E. 1973. Modificaciones al sistema de clasificación climática de Kõeppen (para adaptarlo a las condiciones de la República Mexicana) (No. QC 981. G37 1973). 97 p.

García-Mendoza, A. 1995. Riqueza y endemismo de la familia Agavaceae en México. In: Conservación de Plantas en Peligro de Extinción: diferentes enfoques. Linares, P.; Dávila. F.; Chiang, R.; Bye, T. (comps). Instituto de Biología, UNAM. México. DF. 51-75 pp.

García-Mendoza, A. J. 2011. Flora del Valle de Tehuacán-Cuicatlán. Agavaceae. Instituto de Biología, Universidad Nacional Autónoma de México, México, D. F. Fascículo. 88:1-95.

Gaspar, R. H. F. y Pérez, A. C. E. 2009. Caracterización morfológica y patrones de distribución de una población nativa de pitayo [Stenocereus griseus (Haworth)] en el municipio de San Ciro de Acosta, SLP. Universidad Autónoma Chapingo, Chapingo, México. 70 p.

Gentry, A. H. 1982. Neotropical floristic diversity: phytogeographical connections between Central and South America, Pleistocene climatic fluctuations, or an accident of the Andean orogeny? Annals of the Missouri Botanical Garden. 69(3):557-593.

Godoy, A.; Herrera, T. y Ulloa, M. 2003. Más allá del pulque y del tepache, las bebidas alcohólicas no destiladas indígenas de México. Primera edición. Instituto de Investigaciones Antropológicas, UNAM. México. 107 p.

Gutiérrez, C. M. C.; Ortiz, S. C. A; Fernández, G. B. S.; Gutiérrez, C. E. V. y González, V. T. 2017. Los suelos del área de influencia del Códice Santa María Asunción y su representación pictórica. Terra Latinoamericana. 35(2):101-111. 
Instituto Nacional de Estadística y Geografía 2010. Compendium de información geográfica municipal. Tepetlaoxotoc, México. 32 p.

Koohafkan, P. and Altieri, M. A. 2011. Globally important agricultural heritage systems: a legacy for the future. Food and Agriculture Organization of the United Nations. Rome. 41 p.

Montemayor, C. 2009. Diccionario del náhuatl en el español de México: nueva edición corregida y aumentada. Gobierno del Distrito Federal y Universidad Nacional Autónoma de México. México, DF. 440 p.

Mora-López, J. L.; Reyes-Agüero, J. A.; Flores-Flores, J. L.; Peña-Valdivia, C. B. y AguirreRivera, J. R. 2011. Variación morfológica y humanización de la sección Salmianae del género Agave. Agrociencia. 45(4):465-477.

Olivier, G. 2012. Los dioses ebrios del México antiguo: de la transgresión a la inmortalidad. Arqueología Mexicana. XIX(114):26-33.

Rzedowski, G. C.; Rzedowski, J. 2005. Flora fanerogámica del Valle de México. $2^{\text {da. }}$ Ed. Instituto de Ecología y Comisión Nacional para el Conocimiento y Uso de la Biodiversidad. Pátzcuaro Michoacán. 1406 p.

Secretaría de Agricultura, Ganadería, Desarrollo Rural, Pesca y Alimentación y Servicio Nacional de Inspección y Certificación de Semillas. 2014. Guía Técnica para la descripción varietal de Agave spp. México DF. 25 p.

Valadez, M. M. J. 2014. "Pulque limpio" / "Pulque sucio": disputas en torno a la legitimidad y la producción social del valor. Revista Colombiana de Antropología. 50(2):41-63.

Vázquez, G. A.; Aliphat, F. M. M.; Estrella, Ch. N. G.; Ortiz, T. E.; Ramírez, J. J. y Ramírez, A. M. 2016. El maguey pulquero, una planta multifuncional y polifacética: los usos desde una visión mestiza e indígena. Scripta Ethnologica. 38(1):65-87.

Vela, E. 2018. Un don divino. El pulque. Arqueología Mexicana. Edición especial. 78:48-75. 